\title{
Polymorphisms near EXOC4 and LRGUK on chromosome 7q32 are associated with Type 2 Diabetes and fasting glucose; The NHLBI Family Heart Study
}

\author{
Jason M Laramie* ${ }^{* 1,2}$, Jemma B Wilk ${ }^{\dagger 1,3}$, Sally L Williamson ${ }^{1}$, \\ Michael W Nagle ${ }^{1}$, Jeanne C Latourelle1, Jennifer E Tobin ${ }^{1,4}$, \\ Michael A Province ${ }^{5}$, Ingrid B Borecki ${ }^{5}$ and Richard H Myers ${ }^{1,2,3}$
}

\begin{abstract}
Address: ${ }^{1}$ Department of Neurology, Boston University School of Medicine, Boston, MA, USA, ${ }^{2}$ Bioinformatics Program, Boston University, Boston, MA, USA, ${ }^{3}$ The Section of Preventive Medicine and Epidemiology, Department of Medicine, Boston University School of Medicine, Boston, MA, USA, ${ }^{4}$ Department of Anatomy and Neurobiology, Boston University School of Medicine, Boston, MA, USA and ${ }^{5}$ Center for Human Genome Sciences, Washington University School of Medicine, St. Louis, MO, USA

Email: Jason M Laramie* - laramiej@gmail.com; Jemma B Wilk - jwilk@bu.edu; Sally L Williamson - sallyw@bu.edu; MichaelW Nagle - mwn@bu.edu; Jeanne C Latourelle - jlatoure@bu.edu; Jennifer E Tobin - jetobin@bu.edu; Michael A Province - mike@dsgmail.wustl.edu; Ingrid B Borecki - ingrid@dsgmail.wustl.edu; Richard H Myers - rmyers@bu.edu

* Corresponding author †Equal contributors
\end{abstract}

Published: 22 May 2008

BMC Medical Genetics 2008, 9:46 doi:10.1 186/147I-2350-9-46

This article is available from: http://www.biomedcentral.com/I47I-2350/9/46

(c) 2008 Laramie et al; licensee BioMed Central Ltd.

This is an Open Access article distributed under the terms of the Creative Commons Attribution License (http://creativecommons.org/licenses/by/2.0), which permits unrestricted use, distribution, and reproduction in any medium, provided the original work is properly cited.
Received: 17 January 2008

Accepted: 22 May 2008

\begin{abstract}
Background: The chromosome 7q32 region is linked to metabolic syndrome and obesity related traits in the Family Heart Study. As part of a fine mapping study of the region, we evaluated the relationship of polymorphisms to fasting glucose levels and Type 2 diabetes.

Methods: Thirty-nine HapMap defined tag SNPs in a $1.08 \mathrm{Mb}$ region and a novel deletion polymorphism were genotyped in 2,603 participants of the NHLBI Family Heart Study (FHS). Regression modeling, adjusting for BMI, age, sex, smoking and the TCF7L2 polymorphism, was used to evaluate the association of these polymorphisms with T2D and fasting glucoses levels.

Results: The deletion polymorphism confers a protective effect for T2D, with homozygous deletion carriers having a 53\% reduced risk compared to non-deleted carriers. Among nondiabetics, the deletion was significantly associated with lower fasting glucose levels in men $(p=$ 0.038 ) but not women ( $p=0.118)$. In addition, seven SNPs near the deletion were significantly associated $(p<0.01)$ to diabetes.

Conclusion: Chromosome 7q32 contains both SNPs and a deletion that were associated to T2D. Although the deletion region contains several islands of strongly conserved sequence, it is not known to contain a transcribed gene. The closest nearby gene, EXOC4, is involved in insulinstimulated glucose transport and may be a candidate for this association. Further work is needed to determine if the deletion represents a functional variant or may be in linkage disequilibrium with a functional mutation influencing EXOC4 or another nearby gene.
\end{abstract}




\section{Background}

Type 2 diabetes (T2D) is characterized by hyperglycemia due to insulin resistance and is accompanied by a failure of $\beta$ cells to produce sufficient insulin. In the United States, the prevalence of diabetes has risen $40 \%$ from 1990 to 1999 [1]. This increase in T2D has been attributed to the recent rise in obesity. The correlation between diabetes and obesity has been shown in numerous epidemiological studies [2,3] although the mechanisms underlying this phenomenon are largely unknown. One current hypothesis proposes that malnutrition in the fetus can lead to the developmental impairment of pancreatic $\beta$ cells, termed a "thrifty phenotype" and that later in life, these children are more susceptible to diabetes [4].

Candidate gene studies in T2D have implicated numerous gene variants that decrease disease risk, such as PPARG (P12A) [5], and others that increase disease risk, such as KCNJ11(E23K) [6]. One of the strongest T2D-associated loci has been mapped to the transcription factor TCF7L2 [7], (odds ratio $\approx 1.7$ ) and this association has been replicated in numerous subsequent studies [8-20].

In addition to single nucleotide polymorphisms (SNPs), genomic insertion/deletion polymorphisms may also influence disease risk. One such example is the deletion polymorphism in the angiotension-converting enzyme (ACE), which has been shown to confer increased risk of coronary artery disease [21]. Recently the HapMap genotype data was used to search for segregating deletions by examining physically clustered failed SNP genotype assays, Mendelian inconsistencies, and departures from Hardy-Weinberg disequilibrium [22]. Five hundred and forty-one deletions were identified ranging in size from 1 to $754 \mathrm{~kb}$. One predicted $10.3 \mathrm{~kb}$ deletion polymorphism was located under a widely replicated obesity linkage peak on chromosome 7q22-q36 [23-32] between the EXOC4 and LRGUK genes. Importantly, linkage to metabolic syndrome has also been reported for the Family Heart Study cohort in the 7q32 region [33].

The aim of this study was to examine SNPs and a chromosomal deletion on chromosome $7 \mathrm{q} 32$ in a sample of families exhibiting linkage to obesity and metabolic syndrome in the region $[27,33]$. Due to the presence of a gene (EXOC4) that is part of the exocyst complex (Exo70), which is involved in insulin-stimulated glucose transport, association to T2D risk and blood glucose levels was hypothesized. In addition, we examined the association between the minor allele of the SNP rs7903146 [7] within the transcription factor TCF7L2 and T2D disease risk. Each polymorphism's association to T2D risk was examined in a large Caucasian subset of the Family Heart Study (FHS) population comprising 2,396 participants (205 T2D cases). In addition to T2D disease affection status, fasting plasma glucose (FPG) was also examined among non-diabetics.

\section{Methods \\ Subjects}

The National Heart, Lung and Blood Institute (NHLBI) Family Heart Study (FHS) recruited families from four existing study centers located in Forsyth County, NC; Framingham, MA; Minneapolis, MN; and Salt Lake City, UT. Approximately one half of the families recruited from these study centers were at high risk for coronary heart disease (CHD) while the other half were selected randomly from their respective study populations.

The body mass index (BMI) SNP fine mapping study genotyped 2,421 FHS participants including 158 diabetics. For the deletion study, 416 of these, including two diabetics, either did not genotype for the deletion or did not have sufficient DNA for the deletion study. Therefore, an additional 182 FHS samples, including 49 diabetics, not originally included in the SNP analysis were typed for the deletion. In total, 2,603 participants, including 207 diabetics, were studied.

T2D diabetes was defined by self-report of diabetes diagnosis and limited to those with an age at diagnosis greater than 25. The controls used were participants recruited as part of FHS who did not report a diagnosis of diabetes. An enzymatic (glucose-oxidase) method (Kodak Ektachem 700 Analyzer, Rochester, NY) was used to measure fasting serum glucose as $\mathrm{mg} / \mathrm{dL}$. This study was approved by the institutional review boards (IRB) of the participating institutions and appropriate informed consent was obtained.

\section{Deletion Detection}

Deletions were detected in the study population using real-time polymerase chain reaction (RT-PCR). To accurately type deletion variants, we designed primers to amplify regions within the proposed chromosomal deletion on chromosome 7q32. We performed TaqMan RTPCR assays, using a VIC-labeled probe for a known diploid gene PMP22 (NM_153321) as a control reference and a VIC-labeled probe (Applied Biosystems, Foster City CA) for the experimental region, each run simultaneously. Each DNA sample was run in quadruplicate for each TaqMan assay on the PRISM ${ }^{\circledast} 7900$ HT Sequence Detection System. The cycles-to-threshold $\left(\mathrm{C}_{\mathrm{t}}\right)$ was determined for each assay separately, and the difference between the average $\mathrm{Ct}$ for the experimental probe and control assays $\left(\Delta \mathrm{C}_{\mathrm{t}}\right)$ was used to infer the presence of zero, one or two copies of the deleted segment. For the examined FHS study sample, the average $\Delta \mathrm{C}_{\mathrm{t}}$ values clustered into three discrete groups, including one group showing amplification of the control locus and no amplification of the experimental locus. Treating each genotype cluster as '+/+' (wildtype), 
'+/-' and '-/-'DNA samples could be assigned a standard genotype (i.e. 11, 12, 22). A small number of individuals whose $\Delta \mathrm{C}_{\mathrm{t}}$ value fell outside of the three genotype clusters $(n=69,2.8 \%)$ were coded as missing genotypes.

\section{SNP Genotyping}

SNPs in the genes neighboring the deletion were genotyped as part of a fine-mapping study of BMI. Thirty-nine Tag SNPs were selected using the HapMap tagger algorithm in the region between 132,552,341 (rs6467475) and 133,619,534 (rs1421483). In addition, the SNP rs7903146, located on chromosome 10 at $114,748,089$ bp within an intron of the gene TCF7L2 (NM_030756), was genotyped. The TCF7L2 SNP was typed using the TaqMan ${ }^{\circledast}$ technology developed by Applied Biosystems (Foster City, CA) using the PRISM ${ }^{\circledast} 7900$ HT Sequence Detection System. The 39 HapMap derived SNPs were genotyped using the Illumina Golden Gate ${ }^{\circledast}$ assay method, through the Illumina Fast-Track Genotyping service. Mendelian inconsistencies were identified using INFER within the PEDSYS software package [34], and genotypes in the pedigrees where inconsistencies were found were removed.

\section{Linkage Disequilibrium (LD) Assessment}

We assessed the LD between the chromosomal deletion obtained by RT-PCR, using deletion genotypes ('+/+', '+/', '-/-') coded as 11, 12 and 22 and adjacent SNPs. The software program Haploview [35] was used to estimate the pairwise LD $\left(r^{2}\right)$ between the chromosomal deletion and nearby SNPs within $500 \mathrm{~kb}$.

\section{Statistical Analysis}

To evaluate the relationship of polymorphisms to T2D, dominant and recessive modeling of the minor allele was performed in a logistic regression implemented with a generalized estimating equation and adjusted for TCF7L2 minor allele, BMI at age 25 (based on participants' self report), study center, age, age ${ }^{2}$, age ${ }^{3}$, sex, and smoking history (never/ever). The relationship to T2D for the TCF7L2 SNP (rs7903146) was modeled as a dominant genetic effect (major homozygotes $=0$, heterozygotes and minor homozygotes $=1$ ) as previously reported [7] and the deletion polymorphism modeled as a recessive genetic effect $('+/+$ wildtype' and '+/-' = 0 and '-/-' = 1) using the same covariates described above. As the FHS sample included prevalent diabetics, analyses of diabetes status were adjusted for an age variable that was defined as the age at diagnosis for diabetic cases and the age at clinical examination for non-diabetic controls. No correction for multiple testing was used in these analyses and, therefore, all pvalues are reported as nominal p-values.

In addition to diabetes status, fasting glucose $(\mathrm{mg} / \mathrm{dL})$ levels were analyzed in non-diabetics using a linear GEE regression model adjusted for BMI, study center, age, age ${ }^{2}$, age $^{3}$, smoking history status and sex. The deletion and TCF7L2 polymorphism were analyzed together in the same model. In this regression model, measurements of $\mathrm{BMI}$ and age at the time of examination were used.

\section{Results}

Clinical characteristics of the study subjects are shown in Table 1 . The mean age at examination of the diabetics was 61.57 with a range from 25.6 to 84.7 and of the non-diabetics participant's mean age was 52.0 with a range from 25.2 to 91.0. Both the BMI at examination $\left(p=10^{-4}\right)$ and the reported BMI at age $25\left(p<10^{-4}\right)$ were significantly different between diabetics and non-diabetics. However, the age at examination of non-diabetics was not different from the age at diagnosis of diabetes among the diabetics $(p=0.82)$.

Table I: Characteristics of the study population

\begin{tabular}{|c|c|c|}
\hline & Diabetics & Non-diabetics \\
\hline$N$ genotyped for deletion & 205 & 1982 \\
\hline$N$ genotyped for SNPs & 158 & 2263 \\
\hline Total studied for either SNPs or deletion* & 207 & 2396 \\
\hline Male & $55.1 \%$ & $46.7 \%$ \\
\hline Age at onset of diabetes (years) & $51.7 \pm 11.6$ & - \\
\hline Age at examination (years) & $62.2 \pm 9.9$ & $51.8 \pm 13.4^{* *}$ \\
\hline BMI $\left(\mathrm{kg} / \mathrm{m}^{2}\right)$ at examination & $30.0 \pm 5.1$ & $27.5 \pm 5.8 * *$ \\
\hline BMI $\left(\mathrm{kg} / \mathrm{m}^{2}\right)$ at age 25 & $24.5 \pm 4.9$ & $22.9 \pm 3.6 * *$ \\
\hline Fasting glucose $(\mathrm{mg} / \mathrm{dL})$ & $173.9 \pm 70.1$ & $95.4 \pm 16.1^{* *}$ \\
\hline \multicolumn{3}{|l|}{ Center (\%): } \\
\hline Forsyth County, North Carolina, & 22.2 & 22.5 \\
\hline Minneapolis, Minnesota & 24.7 & 28.0 \\
\hline Framingham, Massachusetts & 19.8 & 22.0 \\
\hline Salt Lake City, Utah & 33.3 & 27.5 \\
\hline
\end{tabular}

\footnotetext{
*means and frequencies correspond to sample with either deletion or SNP genotyping
} $* * \mathrm{p}<10^{-4}$ 
Table 2: Primer and probe sequences used in the RT-PCR deletion assay

\begin{tabular}{|c|c|c|c|}
\hline Gene & Region & & \\
\hline \multirow[t]{8}{*}{ PMP22 (Control) } & chr|7:15,074,94I-15,075,005 & & \\
\hline & & Primer I & CССTTCTCAGCGGTGTCATC \\
\hline & & Primer 2 & ACAGACCGTCTGGGCGC \\
\hline & & Probe & VIC - TTCGCGTTTCCGCAAGAT \\
\hline & chr7:133,44I,108-133,44I,133 & & \\
\hline & & Primer I & GCCTTGCCCGAGTACATATT \\
\hline & & Primer 2 & AGAGTTGGCCTCTGTCCСTA \\
\hline & & Probe & VIC-CAGCTGGTGTTACCAGTAAAGGCCCT \\
\hline
\end{tabular}

\section{Deletion Polymorphism}

Table 2 lists the sequences for the control (PMP22) and deletion detection primers and probes. A total of 2,198 study participants were assayed and the average $\Delta \mathrm{C}_{\mathrm{t}}$ was used to generate three genotype clusters (Figure 1). The deletion was present in $52.7 \%(n=1,152)$ of the study population with a deletion allele frequency of $31.2 \%$ and was in Hardy-Weinberg equilibrium $(p=0.85)$.

Diabetes risk results for the deletion versus the TCF7L2 polymorphism are shown in Table 3 . In the total study sample, there was a protective effect of the homozygous deletion genotype on diabetes ( $p=0.016$ odds ratio (OR) $=0.47,95 \%$ CI $0.25-0.87$ ). In addition, BMI at age 25 was shown to be a strong risk factor for diabetes $\left(p<10^{-4}\right.$, beta estimate $=.09$, OR for a 1 unit BMI increase at age $25=$ $1.09)$. In the total study sample, $5.4 \%(n=11)$ of the diabetics were homozygous for the deletion as compared to $10.2 \%(n=203)$ of the non-diabetics.

When stratified by sex, the protective effect of the deletion on diabetes was stronger in men $(p=0.064, \mathrm{OR}=0.45$,

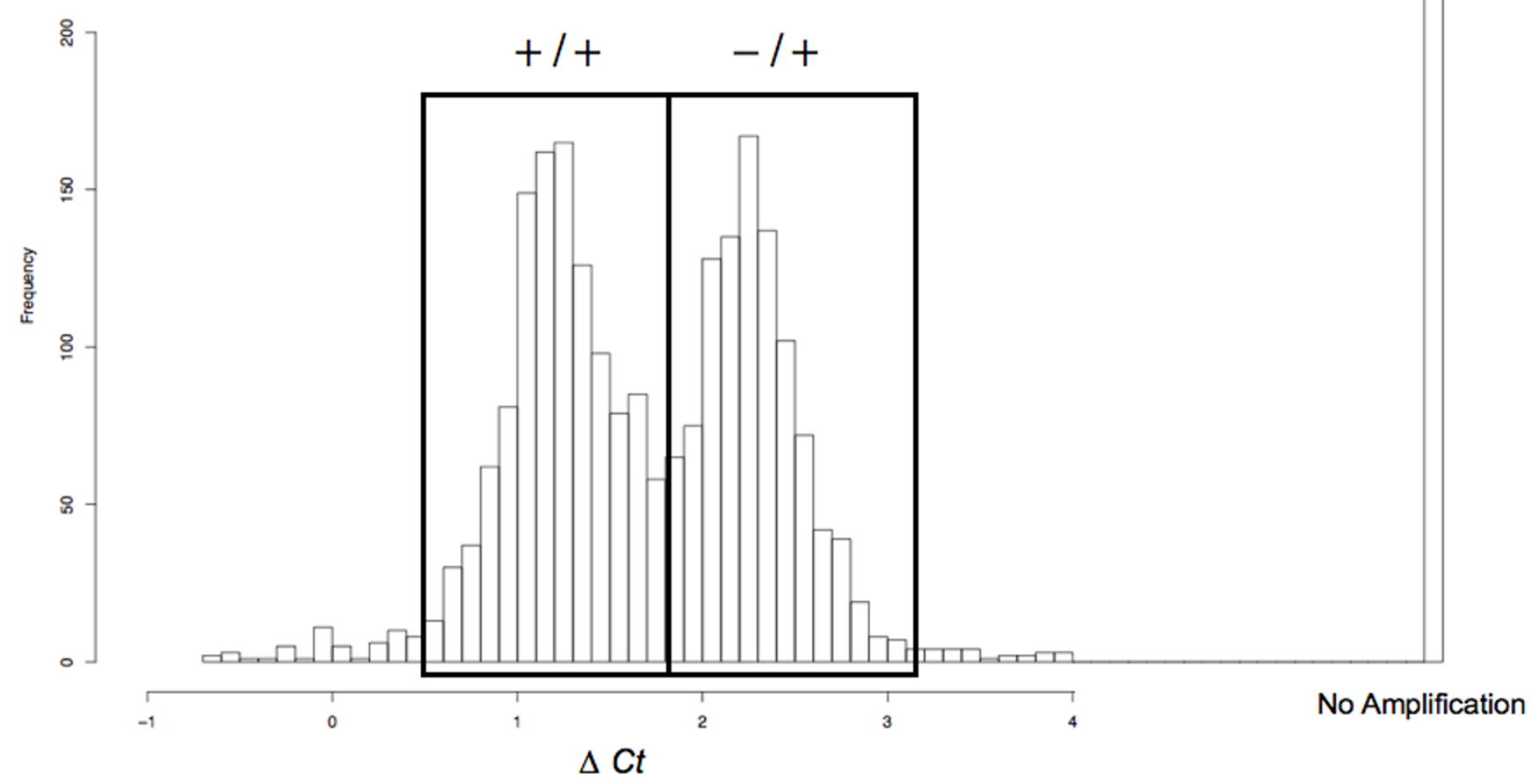

Figure I

Insertion/deletion genotype clusters. Deletion genotype clusters as measured by real-time PCR. Individuals outside of the clusters, defined by the black boxes, were marked with an unknown genotype. 
Table 3: Logistic GEE results incorporating both polymorphisms

\begin{tabular}{|c|c|c|c|c|}
\hline \multicolumn{5}{|c|}{ Diabetes Status } \\
\hline & \multicolumn{2}{|c|}{ Homozygous deletion } & \multicolumn{2}{|c|}{ rs7903 I46 minor allele } \\
\hline & OR $[95 \% \mathrm{Cl}]$ & p-value & OR $[95 \% \mathrm{Cl}]$ & $p$-value \\
\hline Both sexes & $0.47[0.25,0.87]$ & 0.016 & $1.31[0.95,1.81]$ & 0.099 \\
\hline Women & $0.57[0.22,1.52]$ & 0.26 & $1.63[1.02,2.59]$ & 0.039 \\
\hline Men & $0.45[0.20,1.05]$ & 0.064 & $1.14[0.76,1.72]$ & 0.53 \\
\hline
\end{tabular}

Results from logistic GEE models incorporating the deletion genotyping and rs7903।46, including sex-stratified results. Odds ratios (OR), $95 \%$ confidence interval $(95 \% \mathrm{Cl})$ and $p$-values are reported.

*205 cases, 1982 controls - adjusted for BMI at age25, onset age for cases ( $w / s q u a r e$ and cubed)

95\% CI 0.2-1.05) than in women $(p=0.26, \mathrm{OR}=0.57$, $95 \%$ CI $0.22-1.52$ ) with BMI at age 25 remaining a strong risk factor for diabetes in each gender $\left(p<10^{-4}\right)$. In men, $5.4 \%(n=6)$ of the diabetics were homozygous for the deletion as compared to $11.4 \%(n=107)$ of the non-diabetics. In women, the individuals homozygous for the deletion represented $5.4 \%(n=5)$ of the diabetics and $9.2 \%(n=96)$ of the non-diabetics, although the effect was not statistically significant.

In addition, we tested the effect of the deletion and the TCF7L2 SNP on fasting glucose levels among non-diabetics stratified by sex (see Table 4). In an analysis of both sexes combined, the TCF7L2 minor allele was associated with a $1.3 \mathrm{mg} / \mathrm{dL}$ higher mean glucose level $(\mathrm{p}=0.045)$, whereas the deletion polymorphism did not have a significant effect on glucose levels. Men homozygous for the deletion polymorphism had a statistically significant decrease in fasting glucose levels $(p=0.038$, $\beta$-estimate $=$ $-2.57 \mathrm{mg} / \mathrm{dL}$ ) while the TCF7L2 SNP had a modest increase in fasting glucose levels $(p=0.089$, $\beta$-estimate $=$ $1.91 \mathrm{mg} / \mathrm{dL}$ ). Neither polymorphism had a significant effect on fasting glucose in women.

\section{SNP Association}

Association analyses to diabetes using SNPs in the EXOC4 and LRGUK gene regions identified multiple polymor-

Table 4: GEE results for fasting glucose levels (mg/dl)

\begin{tabular}{|c|c|c|c|c|}
\hline & \multicolumn{2}{|c|}{ Homozygous deletion } & \multicolumn{2}{|c|}{ rs7903 I 46 minor allele } \\
\hline & beta-estimate & p-value & Beta-estimate & $p$-value \\
\hline Both sexes & -0.50 & 0.57 & 1.33 & 0.045 \\
\hline Women & 1.89 & 0.118 & 0.77 & 0.283 \\
\hline Men & -2.57 & 0.038 & 1.91 & 0.089 \\
\hline
\end{tabular}

Results from GEE models for the deletion genotyping and rs7903।46 modeled together including sex-stratified results. phisms with evidence for association (Table 5). Seven SNPs (rs3823572, rs12531707, rs11770757, rs7457999, rs6953590, rs12670589, and rs1421483) demonstrated significant association $(\mathrm{p}=0.01)$ to fasting glucose. Using a dominant modeling of the minor allele, the SNP rs 12531707 in an EXOC4 intron produced an odds ratio for diabetes of $1.79(\mathrm{p}=0.009)$. The SNP in strongest LD with the deletion, rs7457999, exhibited a protective effect for diabetes. Using a recessive modeling of the minor allele, the SNP rs12670589 in a $L R G U K$ intron produced an OR for diabetes of $2.02(\mathrm{p}=0.002)$. Other SNPs in both EXOC4 and LRGUK produced larger ORs for the recessive model, but the results were based on a small number of homozygous minor allele carriers. For example, rs11770757 produced an OR $=11.9$ based on two homozygous minor allele carriers each in cases and controls, and the results may be spurious. We have presented all recessive results for which the model converged in the hopes that replication studies in larger samples of diabetics will examine these SNPs.

Within the total study population the SNP rs7903146, located within an intron of the gene TCF7L2 (NM_030756), had a minor allele frequency of $31 \%$. In a dominant model, the TCF7L2 SNP was modestly associated with diabetes risk ( $p=0.099, \mathrm{OR}=1.31,95 \% \mathrm{CI}$ $0.95-1.81)$. In the total study sample, $57.5 \%(n=119)$ of the diabetics were minor risk allele carriers as compared to $51.9 \%(n=1243)$ of the non-diabetics.

When stratified by sex, the minor allele of the TCF7L2 SNP was associated with a large increased risk for T2D in women $(p=0.039, \mathrm{OR}=1.63,95 \%$ CI $1.02-2.59)$ whereas no effect was seen in men $(p=0.53, \mathrm{OR}=1.14$, $95 \%$ CI $0.76-1.72)$. In women, $63.4 \%(n=59)$ of the diabetics were minor allele risk carriers compared to $50.6 \%$ $(n=646)$ of the non-diabetics. In men, $52.6 \%(n=60)$ of the diabetics were minor allele risk carriers compared to $52.6 \%(n=597)$ of the non-diabetics.

Finally, the linkage disequilibrium $\left(r^{2}\right)$ between the deletion polymorphism and the thirty-nine surrounding SNPs genotyped in the total study population is displayed in Figure 2 . The deletion polymorphism exhibited modest LD with SNP rs7457999 $\left(r^{2}=0.37\right)$ and $\operatorname{rs} 13246630\left(r^{2}=\right.$ $0.20)$.

\section{Discussion}

The rs7903146 SNP in the TCF7L2 gene represents perhaps the most important gene polymorphism implicated in type 2 diabetes, since it is a relatively common variant that confers increased risk for diabetes and this association has been replicated across numerous independent samples $[7,9,10,13,17,20,36]$. In this study, we report a novel deletion polymorphism on chromosome $7 \mathrm{q} 32$ that 
Table 5: SNP association to diabetes in region surrounding deletion

\begin{tabular}{|c|c|c|c|c|c|c|}
\hline SNP & bp position & Gene & Dominant OR & Dominant $p$-value & Recessive OR & Recessive $p$-value \\
\hline rs6467475 & |3255234| & & 1.04 & 0.85 & 1.29 & 0.30 \\
\hline rs|l 979455 & $|32558| 9 \mid$ & & 1.22 & 0.24 & 1.46 & 0.20 \\
\hline rs6979285 & | 32564427 & & 0.70 & 0.22 & & \\
\hline rs6951889 & 132566505 & & 0.73 & 0.09 & 0.90 & 0.77 \\
\hline rs 10262862 & | 32606574 & EXOC4 & 1.03 & 0.88 & 1.36 & 0.19 \\
\hline rs|3242614 & 132616839 & EXOC4 & 0.93 & 0.76 & 2.67 & 0.03 \\
\hline rs 1922420 & 132647772 & EXOC4 & 0.94 & 0.74 & 1.07 & 0.86 \\
\hline rs6978272 & | 32738579 & EXOC4 & 1.03 & 0.88 & 0.76 & 0.71 \\
\hline rs|324||23 & | 32786047 & EXOC4 & 0.83 & 0.29 & 0.79 & 0.42 \\
\hline rs697|417 & 132816709 & EXOC4 & 1.45 & 0.04 & 1.42 & 0.33 \\
\hline rs 13237737 & 132842959 & EXOC4 & 1.15 & 0.71 & 4.00 & 0.18 \\
\hline rs 10755879 & 132910055 & EXOC4 & 0.71 & 0.14 & 2.10 & 0.17 \\
\hline rs6954842 & | 32938673 & EXOC4|KIAAI699 & 1.19 & 0.35 & 1.23 & 0.42 \\
\hline rs 1362736 & 133060409 & EXOC4|KIAA I699 & 1.00 & 1.00 & 1.59 & 0.47 \\
\hline rs17167240 & 133075208 & EXOC4|KIAAI699 & 0.83 & 0.34 & 0.44 & 0.11 \\
\hline rs9649047 & 133084054 & EXOC4|KIAAI699 & 0.98 & 0.93 & 1.26 & 0.72 \\
\hline rs|l 772444 & $1331044 \mid 4$ & EXOC4|KIAAI699 & 1.51 & 0.02 & 1.72 & 0.11 \\
\hline rs I3222377 & 133116886 & EXOC4|KIAAI 699 & 0.99 & 0.97 & 0.89 & 0.79 \\
\hline rs 17167267 & 133119649 & EXOC4|KIAA I 699 & 1.15 & 0.56 & & \\
\hline rs748754 & 133159609 & EXOC4|KIAAI699 & 1.09 & 0.60 & 0.81 & 0.52 \\
\hline rs/2155007 & 133235969 & EXOC4|KIAAI699 & 0.74 & 0.31 & 4.79 & 0.05 \\
\hline rs 4266574 & | 33277976 & EXOC4|KIAA I 699 & 0.80 & 0.24 & 0.29 & 0.04 \\
\hline rs297|970 & $1332943 \mid 8$ & EXOC4|KIAAI699 & 1.42 & 0.07 & 1.48 & 0.25 \\
\hline rs3823572 & $|3333||4|$ & EXOC4|KIAAI 699 & 0.89 & 0.53 & 0.58 & 0.01 \\
\hline rs| 2531707 & |3337877| & EXOC4|KIAA I699 & 1.79 & 0.009 & 1.15 & 0.49 \\
\hline rs6955II4 & | 33387570 & EXOC4|KIAA I 699 & 1.26 & 0.20 & 0.97 & 0.90 \\
\hline rs6971064 & 133390804 & EXOC4|KIAA I699 & 1.17 & 0.40 & 0.87 & 0.81 \\
\hline rsII 770757 & I 33398486 & EXOC4|KIAA I 699 & 0.71 & 0.33 & 11.87 & 0.006 \\
\hline rs7457999 & 133398775 & EXOC4|KIAAI 699 & 0.62 & 0.01 & 0.58 & 0.22 \\
\hline rs 10246346 & $|334| 586 \mid$ & & 1.04 & 0.86 & 0.79 & 0.76 \\
\hline \multicolumn{7}{|l|}{ Deletion } \\
\hline rs 13246630 & 133472159 & LRGUK & 1.36 & 0.09 & 1.27 & 0.27 \\
\hline rs6953590 & 133487840 & LRGUK & 1.52 & 0.01 & 1.86 & 0.006 \\
\hline rs|7761994 & 133494082 & LRGUK & 1.23 & 0.35 & & \\
\hline rs| 2670589 & 133495637 & LRGUK & 1.30 & 0.14 & 2.03 & 0.002 \\
\hline rs892984 & $|3350272|$ & LRGUK & 0.64 & 0.10 & 0.92 & 0.92 \\
\hline rs 1222430 & 133566300 & LRGUK & 0.73 & 0.24 & 1.08 & 0.93 \\
\hline rs|42|477 & 133569572 & LRGUK & 1.15 & 0.51 & & \\
\hline rs 1450890 & | 33584737 & LRGUK & 0.86 & 0.41 & 1.31 & 0.33 \\
\hline rs| $42 \mid 483$ & 133619284 & & 1.01 & 0.97 & 3.93 & 0.009 \\
\hline
\end{tabular}

confers a protective effect for diabetes, with homozygous deletion carriers having a 53\% reduced risk of diabetes compared to carriers of the non-deleted region. In addition, we demonstrate an increased risk associated with the minor allele of the TCF7L2 SNP that is identified primarily in women, but not men of the Family Heart Study. In this sample, the statistical evidence for association to T2D of the homozygous deletion, although it reduces rather than increases risk, was comparable to that of the TCF7L2 SNP. Several nearby SNPs in the 7q32 region also show significant association to $\mathrm{T} 2 \mathrm{D}$, and this may represent linkage disequilibrium among these various polymorphisms. Finally, we report that non-diabetic male homozygous deletion carriers had significantly lower fast- ing glucose levels, suggesting that the risk for T2D may be mediated by reduced glucose levels.

The region implicated by the deletion and SNP polymorphisms reported here is located between the genes EXOC4 (NM_021807) and LRGUK (NM_144648). Interestingly, EXOC4 is a large gene and its product is part of the exocyst complex 70 (Exo70) that assembles at the plasma membrane of adipocytes in response to insulin and has been reported to play a role in docking and tethering the glucose transporter 4 (GLUT4) vesicle to the plasma membrane $[37,38]$. GLUT4 accounts for much of the insulinstimulated glucose transport in muscle and adipose tissue $[38,39]$. Inoue et al. (2003) report variability in insulinstimulated glucose uptake with Exo70 variants, and 


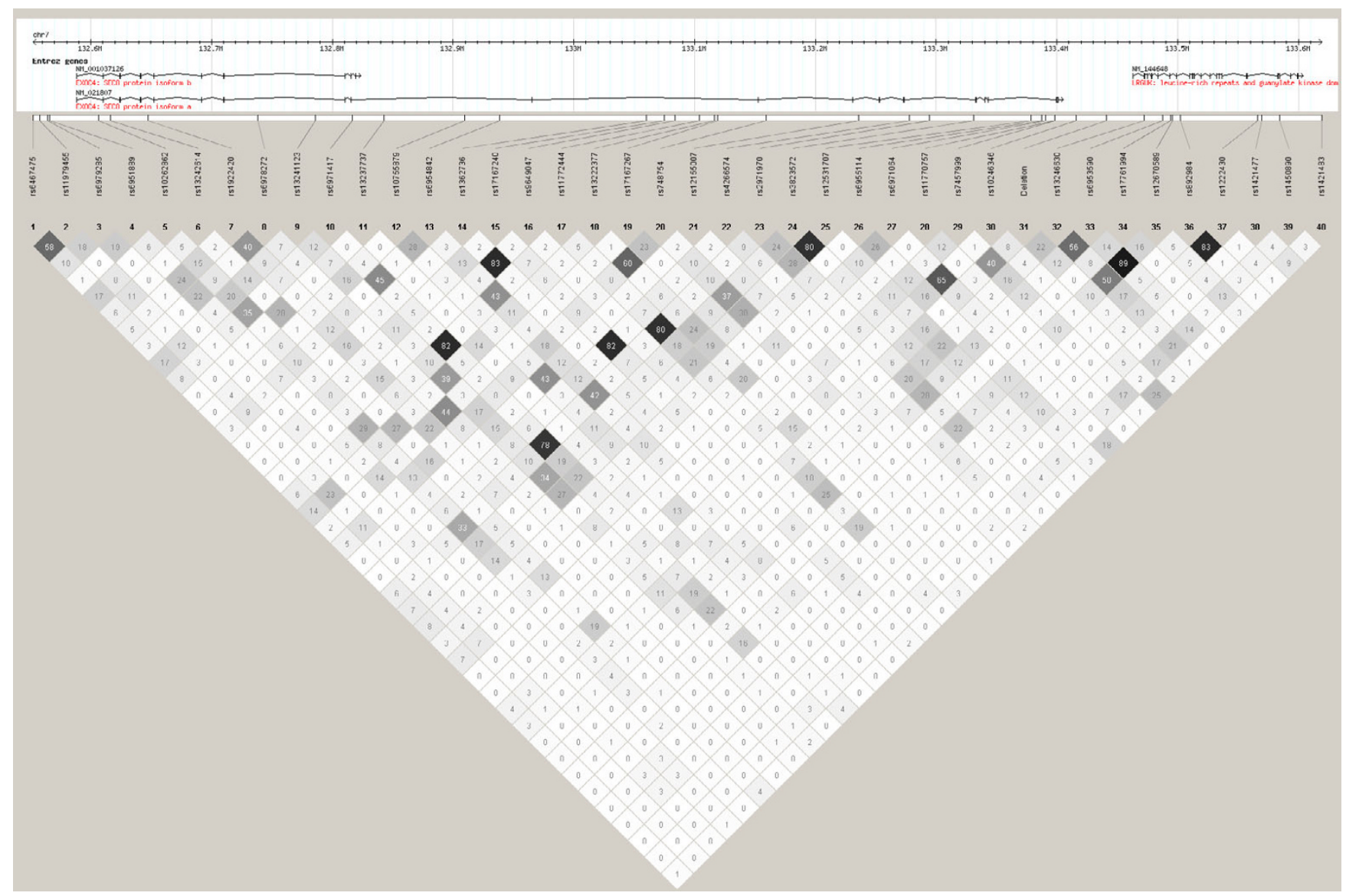

Figure 2

LD structure surrounding the insertion/deletion polymorphism. Linkage disequilibrium $\left(r^{2}\right)$ of the deletion polymorphism ('Deletion') and surrounding genotyped SNPs within the FHS study population. The legend above the LD plot shows the location of the genes EXOC4 and LRGUK (FLJ32786).

EXOC4 was shown to interact with both the Exo70 wildtype and the amino-terminal fragment of Exo70, which may block the insulin-stimulated assembly of exocyst complex at the plasma membrane. EXOC4 has also been shown to be involved the initial docking of insulin vesicles to the cell membrane of pancreatic $\beta$ cells and is thought to play a role in regulating insulin vesicle exocytosis in response to a glucose stimulus [40]. The potential for this gene to influence diabetes and glucose levels prompted us to evaluate the association to these traits. Though the deletion polymorphism does not seem to overlap with EXOC4, transcriptional binding elements could exist downstream of the gene within the region of the deletion that influence transcription. Finally, differences in EXOC4 transcription levels could affect glucose stimulated insulin release as well as insulin induced cellular uptake of glucose resulting in the decreased fasting plasma glucose levels found for homozygous deletion carriers in non-diabetics.
Findings in other cohorts also support the presence of a gene(s) influencing diabetes risk in this region. Genomewide associations for diabetes were recently performed by the Wellcome Trust Case Control Consortium (WTCCC) [41] and the Diabetes Genetics Initiative (DGI) [42], and the results are publicly available. We examined association results for the SNPs in the region spanning the EXOC4 gene, the deletion, and the LRGUK gene, which included 202 SNPs in the WTCCC and 128 SNPs in the DGI studies. From the Wellcome Trust results, we identified 37 SNPs with association p-values less than 0.05 , and from the DGI, we identified ten SNPs with association pvalues less than 0.05 . The best p-value identified in the Wellcome Trust results in this region was 0.0004 at rs6963221 in EXOC4. In the DGI results, the best p-value was 0.015 at rs 17167492 in LRGUK. These results from two independent samples lend support for polymorphisms in the region influencing diabetes risk. 
One limitation of our study is the self-reported diabetes status. Individuals used in our fasting glucose analysis may have been diagnosed with diabetes after FHS study enrollment or may have failed to report themselves as having been diagnosed for diabetes and, therefore, our analysis of fasting glucose may have included undiagnosed diabetics in the non-diabetic study population. Nevertheless, the glucose levels analyzed were unmedicated measurements.

\section{Conclusion}

In this study, we report a large novel deletion polymorphism that is associated with reduced risk for T2D and several SNPs associated with either increased or decreased risk for $\mathrm{T} 2 \mathrm{D}$ on chromosome $7 \mathrm{q} 32$ located within a widely replicated BMI linkage region [23-32]. In addition, we demonstrate that non-diabetic males that are homozygous for the deletion polymorphism have lower levels of fasting glucose, which may contribute to protection from T2D. Furthermore, when examined together, the deletion polymorphism offers an effect, albeit protective, comparable to the widely replicated risk associated with the SNP rs7903146 within the transcription factor TCF7L2. Since these polymorphisms are in an intergenic region, their relation to nearby gene(s) is speculative and further research will need to be conducted to elucidate the mechanism by which they influence risk for T2D. Ultimately, understanding this mechanism(s) could shed light on the poorly understood relationship between obesity and diabetes and may suggest pathways involved in reducing glucose levels and risk for diabetes.

\section{Abbreviations}

NHLBI: National Heart Lung Blood Institute; FHS: Family Heart Study; BMI: Body mass index; T2D: Type 2 diabetes; PPARG: Peroxisome proliferation activated receptor gamma; KCNJ11: Potassium inwardly-rectifying channel J11; TCF7L2: Transcription factor 7-like 2; SNP: Single nucleotide polymorphism; ACE: angiotension-converting enzyme; EXOC4: Exocyst complex component 4; LRGUK: leucine-rich and gaunylate kinase domain; CHD: Coronary heart disease; IRB: institutional review board; PMP22: Peripheral myelin protein 2; $\mathrm{C}_{\mathrm{t}}$ :cycles-to-threshold; DNA: deoxyribonucleic acid; LD: linkage disequilibrium; RT-PCR: Real time Polymerase chain reaction; GEE: General estimating equation; OR: odds ratio; Exo70: exocyst complex 70; GLUT4: glucose transporter 4; WTCCC: Wellcome Trust Case Control Consortium; DGI: Diabetes Genetics Initiative.

\section{Competing interests}

The authors declare that they have no competing interests.

\section{Authors' contributions}

JML and JBW both assisted in the conception of the study, participated in the design of the study, performed statistical analysis and helped to draft the manuscript. SLW carried out molecular genetic analyses for single nucleotide polymorphisms and the evaluation of the size of the deletion. MWN carried out molecular genetic analyses for SNPs and the deletion assay. JCL coordinated the cleaning and management of SNP and deletion polymorphism data. JET performed molecular genetic analyses of the conserved sequence within the deleted region. MAP and IBB conceived the study and participated in its design and coordination. RHM assisted in the conception of the study, participated in its design and coordination, and helped draft the manuscript. All authors read and approved the final manuscript.

\section{Acknowledgements}

This work was supported in part by NIH grants R0I HL6889I-05 and R0I D068336-03. In addition, this research was conducted using the Boston University Linux Cluster for Genetic Analysis (LinGA) funded by the NIH NCRR (National Center for Research Resources) Shared Instrumentation grant (ISIORRI63736-0IAI).

\section{References}

I. Narayan KM, Boyle JP, Thompson TJ, Sorensen SW, Williamson DF: Lifetime risk for diabetes mellitus in the United States. Jama 2003, 290: 1884-1890.

2. Hu FB, Manson JE, Stampfer MJ, Colditz G, Liu S, Solomon CG, Willett WC: Diet, lifestyle, and the risk of type 2 diabetes mellitus in women. N Engl J Med 2001, 345:790-797.

3. Zimmet PZ: Diabetes epidemiology as a tool to trigger diabetes research and care. Diabetologia 1999, 42:499-518.

4. Hales CN, Barker DJ: The thrifty phenotype hypothesis. Br Med Bull 200I, 60:5-20.

5. Altshuler D, Hirschhorn JN, Klannemark M, Lindgren CM, Vohl MC, Nemesh J, Lane CR, Schaffner SF, Bolk S, Brewer C, Tuomi T, Gaudet D, Hudson TJ, Daly M, Groop L, Lander ES: The common PPARgamma Prol2Ala polymorphism is associated with decreased risk of type 2 diabetes. Nat Genet 2000, 26:76-80.

6. Gloyn AL, Weedon MN, Owen KR, Turner MJ, Knight BA, Hitman G, Walker M, Levy JC, Sampson M, Halford S, McCarthy MI, Hattersley AT, Frayling TM: Large-scale association studies of variants in genes encoding the pancreatic beta-cell KATP channel subunits Kir6.2 (KCNJII) and SURI (ABCC8) confirm that the KCNJ I I E23K variant is associated with type 2 diabetes. Diabetes 2003, 52:568-572.

7. Grant SF, Thorleifsson G, Reynisdottir I, Benediktsson R, Manolescu A, Sainz J, Helgason A, Stefansson H, Emilsson V, Helgadottir A, Styrkarsdottir U, Magnusson KP, Walters GB, Palsdottir E, Jonsdottir T, Gudmundsdottir T, Gylfason A, Saemundsdottir J, Wilensky RL, Reilly MP, Rader DJ, Bagger Y, Christiansen C, Gudnason V, Sigurdsson G, Thorsteinsdottir U, Gulcher JR, Kong A, Stefansson K: Variant of transcription factor 7-like 2 (TCF7L2) gene confers risk of type 2 diabetes. Nat Genet 2006, 38:320-323.

8. Cauchi S, Meyre D, Dina C, Choquet H, Samson C, Gallina S, Balkau B, Charpentier G, Pattou F, Stetsyuk V, Scharfmann R, Staels B, Fruhbeck $G$, Froguel $P$ : Transcription factor TCF7L2 genetic study in the French population: expression in human beta-cells and adipose tissue and strong association with type 2 diabetes. Diabetes 2006, 55:2903-2908.

9. Chandak GR, Janipalli CS, Bhaskar S, Kulkarni SR, Mohankrishna P, Hattersley AT, Frayling TM, Yajnik CS: Common variants in the TCF7L2 gene are strongly associated with type 2 diabetes mellitus in the Indian population. Diabetologia 2007, 50:63-67.

10. Damcott CM, Pollin TI, Reinhart LJ, Ott SH, Shen H, Silver KD, Mitchell BD, Shuldiner AR: Polymorphisms in the transcription factor 7-like 2 (TCF7L2) gene are associated with type 2 
diabetes in the Amish: replication and evidence for a role in both insulin secretion and insulin resistance. Diabetes 2006 55:2654-2659.

II. Florez JC, Jablonski KA, Bayley N, Pollin TI, de Bakker PI, Shuldiner AR, Knowler WC, Nathan DM, Altshuler D: TCF7L2 polymorphisms and progression to diabetes in the Diabetes Prevention Program. N Engl J Med 2006, 355:24I-250.

12. Groves CJ, Zeggini E, Minton J, Frayling TM, Weedon MN, Rayner NW, Hitman GA, Walker M, Wiltshire S, Hattersley AT, McCarthy MI: Association analysis of 6,736 U.K. subjects provides replication and confirms TCF7L2 as a type 2 diabetes susceptibility gene with a substantial effect on individual risk. Diabetes 2006, 55:2640-2644.

13. Humphries SE, Gable D, Cooper JA, Ireland H, Stephens JW, Hure S], Li KW, Palmen J, Miller MA, Cappuccio FP, Elkeles R, Godsland I, Miller G], Talmud PJ: Common variants in the TCF7L2 gene and predisposition to type 2 diabetes in UK European Whites, Indian Asians and Afro-Caribbean men and women. J Mol Med 2006, 84: I- I0.

14. Mayans S, Lackovic K, Lindgren P, Ruikka K, Agren A, Eliasson M, Holmberg D: TCF7L2 polymorphisms are associated with type 2 diabetes in northern Sweden. Eur J Hum Genet 2007, I 5:342-346.

15. Parra E, Cameron E, Simmonds L, Valladares A, McKeigue P, Shriver M, Wacher N, Kumate J, Kittles R, Cruz M: Association of TCF7L2 polymorphisms with type 2 diabetes in Mexico City. Clin Genet 2007, 7I:359-366.

16. Saxena R, Gianniny L, Burtt NP, Lyssenko V, Giuducci C, Sjogren M, Florez JC, Almgren P, Isomaa B, Orho-Melander M, Lindblad U, Daly MJ, Tuomi T, Hirschhorn JN, Ardlie KG, Groop LC, Altshuler D: Common single nucleotide polymorphisms in TCF7L2 are reproducibly associated with type 2 diabetes and reduce the insulin response to glucose in nondiabetic individuals. Diabetes 2006, 55:2890-2895.

17. Scott LJ, Bonnycastle LL, Willer C], Sprau AG, Jackson AU, Narisu N Duren WL, Chines PS, Stringham HM, Erdos MR, Valle TT, Tuomilehto J, Bergman RN, Mohlke KL, Collins FS, Boehnke M: Association of transcription factor 7-like 2 (TCF7L2) variants with type 2 diabetes in a Finnish sample. Diabetes 2006, 55:2649-2653.

18. Sladek R, Rocheleau G, Rung J, Dina C, Shen L, Serre D, Boutin P, Vincent D, Belisle A, Hadjadj S, Balkau B, Heude B, Charpentier G, Hudson TJ, Montpetit A, Pshezhetsky AV, Prentki M, Posner BI, Balding DJ, Meyre D, Polychronakos C, Froguel P: A genome-wide association study identifies novel risk loci for type 2 diabetes. Nature 2007, 445:88I-885.

19. van Vliet-Ostaptchouk JV, Shiri-Sverdlov R, Zhernakova A, Strengman E, van Haeften TW, Hofker MH, Wijmenga C: Association of variants of transcription factor 7-like 2 (TCF7L2) with susceptibility to type 2 diabetes in the Dutch Breda cohort. Diabetologia 2007, 50:59-62.

20. Zhang C, Qi L, Hunter DJ, Meigs JB, Manson JE, van Dam RM, Hu FB Variant of transcription factor 7-like 2 (TCF7L2) gene and the risk of type 2 diabetes in large cohorts of U.S. women and men. Diabetes 2006, 55:2645-2648.

21. Peterlin B, Petrovic D, Zorc M, Keber I: Deletion/insertion polymorphism in the angiotension-converting enzyme gene as a risk factor in the Slovenian patients with coronary heart disease. Pflugers Arch 2000, 439:R40-4I.

22. McCarroll SA, Hadnott TN, Perry GH, Sabeti PC, Zody MC, Barrett JC, Dallaire S, Gabriel SB, Lee C, Daly MJ, Altshuler DM: Common deletion polymorphisms in the human genome. Nat Genet 2006, 38:86-92.

23. Arya R, Blangero J, Williams K, Almasy L, Dyer TD, Leach RJ, O'Connell $P$, Stern MP, Duggirala R: Factors of insulin resistance syndrome - related phenotypes are linked to genetic locations on chromosomes 6 and 7 in nondiabetic mexican-americans. Diabetes 2002, 5 I :841-847.

24. Bray MS, Boerwinkle $E$, Hanis CL: Linkage analysis of candidate obesity genes among the Mexican-American population of Starr County, Texas. Genet Epidemiol 1999, 16:397-4II.

25. Clement K, Garner C, Hager J, Philippi A, LeDuc C, Carey A, Harris T], Jury C, Cardon LR, Basdevant A, Demenais F, Guy-Grand B, North $M$, Froguel $P$ : Indication for linkage of the human $O B$ gene region with extreme obesity. Diabetes 1996, 45:687-690.

26. Duggirala R, Stern MP, Mitchell BD, Reinhart LJ, Shipman PA, Uresandi OC, Chung WK, Leibel RL, Hales CN, O'Connell P, Blangero ]: Quantitative variation in obesity-related traits and insulin precursors linked to the OB gene region on human chromosome 7. Am J Hum Genet 1996, 59:694-703.

27. Feitosa MF, Borecki IB, Rich SS, Arnett DK, Sholinsky P, Myers RH, Leppert M, Province MA: Quantitative-trait loci influencing body-mass index reside on chromosomes 7 and I3: the National Heart, Lung, and Blood Institute Family Heart Study. Am J Hum Genet 2002, 70:72-82.

28. Lapsys NM, Furler SM, Moore KR, Nguyen TV, Herzog H, Howard G, Samaras K, Carey DG, Morrison NA, Eisman JA, Chisholm DJ: Relationship of a novel polymorphic marker near the human obese (OB) gene to fat mass in healthy women. Obes Res 1997 , 5:430-433.

29. Li WD, Li D, Wang S, Zhang S, Zhao H, Price RA: Linkage and linkage disequilibrium mapping of genes influencing human obesity in chromosome region 7q22.1-7q35. Diabetes 2003 , 52:|557-|56|.

30. Reed DR, Ding Y, Xu W, Cather C, Green ED, Price RA: Extreme obesity may be linked to markers flanking the human OB gene. Diabetes 1996, 45:691-694.

31. Rutkowski MP, Klanke CA, Su YR, Reif M, Menon AG: Genetic markers at the leptin (OB) locus are not significantly linked to hypertension in African Americans. Hypertension 1998, 31:1230-1234.

32. Wu X, Cooper RS, Borecki I, Hanis C, Bray M, Lewis CE, Zhu X, Kan $D$, Luke A, Curb D: A combined analysis of genomewide linkage scans for body mass index from the National Heart, Lung, and Blood Institute Family Blood Pressure Program. Am J Hum Genet 2002, 70: 1247-1256.

33. Tang W, Miller MB, Rich SS, North KE, Pankow JS, Borecki IB, Myers $\mathrm{RH}$, Hopkins PN, Leppert M, Arnett DK: Linkage analysis of a composite factor for the multiple metabolic syndrome: the National Heart, Lung, and Blood Institute Family Heart Study. Diabetes 2003, 52:2840-2847.

34. PEDSYS [http://www.sfbr.org/pedsys/pedsys.html]

35. Barrett JC, Fry B, Maller J, Daly MJ: Haploview: analysis and visualization of LD and haplotype maps. Bioinformatics 2005 , 2I:263-265

36. Melzer D, Murray A, Hurst AJ, Weedon MN, Bandinelli S, Corsi AM, Ferrucci L, Paolisso G, Guralnik JM, Frayling TM: Effects of the diabetes linked TCF7L2 polymorphism in a representative older population. BMC Med 2006, 4:34.

37. Inoue $M$, Chang L, Hwang J, Chiang SH, Saltiel AR: The exocyst complex is required for targeting of Glut4 to the plasma membrane by insulin. Nature 2003, 422:629-633.

38. Inoue M, Chiang SH, Chang L, Chen XW, Saltiel AR: Compartmentalization of the exocyst complex in lipid rafts controls Glut4 vesicle tethering. Mol Biol Cell 2006, I 7:2303-23 II.

39. Pessin JE, Thurmond DC, Elmendorf JS, Coker KJ, Okada S: Molecular basis of insulin-stimulated GLUT4 vesicle trafficking. Location! Location! Location! J Biol Chem I 999, 274:2593-2596.

40. Tsuboi T, Ravier MA, Xie H, Ewart MA, Gould GW, Baldwin SA, Rutter GA: Mammalian exocyst complex is required for the docking step of insulin vesicle exocytosis. The Journal of biological chemistry 2005, 280:25565-25570.

4I. Genome-wide association study of 14,000 cases of seven common diseases and 3,000 shared controls. Nature 2007 447:66I-678

42. Saxena R, Voight BF, Lyssenko V, Burtt NP, de Bakker PI, Chen H, Roix J], Kathiresan S, Hirschhorn JN, Daly MJ, Hughes TE, Groop L, Altshuler D, Almgren P, Florez JC, Meyer J, Ardlie K, Bengtsson Bostrom K, Isomaa B, Lettre G, Lindblad U, Lyon HN, Melander O, Newton-Cheh C, Nilsson P, Orho-Melander M, Rastam L, Speliotes EK, Taskinen MR, Tuomi T, Guiducci C, et al.: Genome-wide association analysis identifies loci for type 2 diabetes and triglyceride levels. Science 2007, 3 | 6: | 33 |- |336.

\section{Pre-publication history}

The pre-publication history for this paper can be accessed here:

http://www.biomedcentral.com/1471-2350/9/46/prepub 